\title{
A double mutation (G11778A and G12192A) in mitochondrial DNA associated with Leber's hereditary optic neuropathy and cardiomyopathy
}

Received: July 4, 2002 / Accepted: October 28, 2002

\begin{abstract}
We report a male patient with Leber's hereditary optic neuropathy (LHON) and hypertrophic cardiomyopathy. Besides a G11778A mutation in the ND4 gene of the mitochondrial DNA (mtDNA), one of the most common mutations in LHON patients, sequencing of total mtDNA revealed a G12192A mutation in the tRNA (His) gene that was recently noted to be a risk factor for cardiomyopathy. Because no case of LHON presenting with cardiomyopathy has been reported, the present finding suggests that the G12192A mutation caused cardiomyopathy as an additional symptom. In the present case, the double pathogenic mtDNA mutations may be associated either synergistically or concomitantly with two different clinical manifestations.
\end{abstract}

Key words Leber's hereditary optic neuropathy (LHON) · Cardiomyopathy $\cdot$ Mitochondria $\cdot$ DNA $\cdot$ Mutation

\section{Introduction}

Leber's hereditary optic neuropathy (LHON) is a maternally inherited disease characterized by acute or subacute visual impairment in both eyes. The association between LHON and mitochondrial DNA (mtDNA) was first identi-

M. Mimaki $\cdot$ H. Komaki $\cdot$ J. Akanuma $\cdot$ Y. Goto $(\bowtie)$

Department of Mental Retardation and Birth Defect Research, National Institute of Neuroscience, National Center of Neurology and Psychiatry (NCNP), 4-1-1 Ogawahigashi, Kodaira, Tokyo

187-8502, Japan

Tel. +81-42-346-1713; Fax +81-42-346-1743

e-mail: goto@ncnp.go.jp

A. Ikota

Department of Internal Medicine, Kin-ikyo Sapporo Hospital, Sapporo, Japan

A. Sato

Department of Medical Chemistry, Kagawa Nutrition University, Saitama, Japan

I. Nonaka

National Center Hospital for Mental, Nervous and Muscular

Disorders, NCNP, Tokyo, Japan fied in 1988 when a G11778A mutation in the ND4 gene of mtDNA was found in nine unrelated families (Wallace et al. 1988). Since then, it has come to be understood that this is one of the most common mutations causing LHON (Riordan-Eva et al. 1995). It is also recognized that some idiopathic cardiomyopathy is associated with mtDNA mutations (Chan and Allen 1995), and a G12192A mutation in tRNA (His) was recently reported to be a risk factor for cardiomyopathy (Shin et al. 2000).

Here, we report a unique patient with LHON presenting with hypertrophic cardiomyopathy, who carried both the G11778A and G12192A mutations.

\section{Patient and methods}

A 51-year-old Japanese man was admitted to hospital because of subacute body weight loss and general malaise. His past history was unremarkable except for mild diabetes mellitus of 5 years' duration. He smoked 20-40 cigarettes and drank $1000 \mathrm{ml}$ of beer and a third of a bottle of whisky almost every day. On admission, he was suffering from chronic renal failure accompanied by hypertension. A son of his sister's daughter had suffered visual loss since the age of 12 years, but there was no other family history related to the patient's condition.

Six months after he presented at the hospital, he began to experience rapid progressive visual loss. On examination using the Landolt ring visual chart, his visual acuity in the right eye was found to have decreased from 1.2 to 0.01 in only 3 months, and successively it decreased rapidly in the left eye. Finally, he became blind about 6 months later. Examination of the ocular fundus showed peripapillary telangiectasia without findings of diabetic retinopathy. His renal failure was also becoming worse simultaneously with the decrease of visual acuity, and hemodialysis became necessary 2 years after his hospital stay. A renal biopsy revealed pathological findings mimicking those of the end stage of arteriosclerotic kidney without the characteristic findings of diabetic nephropathy. The diabetes mellitus was 
Table 1. MtDNA sequence variants in the patient

\begin{tabular}{|c|c|c|c|c|}
\hline Gene product & Nucleotide number & Base change & Amino acid change & MitoMap database \\
\hline D-loop & 514 & $\mathrm{C}$ to deletion & & Reported polymorphism \\
\hline D-loop & 515 & A to deletion & & Reported polymorphism \\
\hline $12 \mathrm{~S}$ rRNA & 709 & $\mathrm{G}$ to $\mathrm{A}$ & & Reported polymorphism \\
\hline 12S rRNA & 750 & A to $\mathrm{G}$ & & Reported polymorphism \\
\hline $12 \mathrm{~S}$ rRNA & 1598 & $\mathrm{G}$ to $\mathrm{A}$ & & Reported polymorphism \\
\hline $16 \mathrm{~S}$ rRNA & 2706 & A to $G$ & & Reported polymorphism \\
\hline NADH dehydrogenase 2 & 4769 & A to $\mathrm{G}$ & Synonymous & Reported polymorphism \\
\hline Cytochrome c oxydase 1 & 7028 & $\mathrm{C}$ to $\mathrm{T}$ & Synonymous & Reported polymorphism \\
\hline ATP synthase 6 & 8784 & $\mathrm{G}$ to $\mathrm{A}$ & Synonymous & Reported polymorphism \\
\hline ATP synthase 6 & 8829 & $\mathrm{C}$ to $\mathrm{T}$ & Synonymous & Unreported \\
\hline ATP synthase 6 & 8860 & A to $G$ & $\mathrm{~T}$ to $\mathrm{A}$ & Reported polymorphism \\
\hline Cytochrome c oxydase 3 & 9950 & $\mathrm{~T}$ to $\mathrm{C}$ & Synonymous & Reported polymorphism \\
\hline NADH dehydrogenase 4 & 11719 & $\mathrm{G}$ to $\mathrm{A}$ & Synonymous & Reported polymorphism \\
\hline NADH dehydrogenase 4 & 11778 & $\mathrm{G}$ to $\mathrm{A}$ & $\mathrm{R}$ to $\mathrm{H}$ & \\
\hline NADH dehydrogenase 4 & 11914 & $\mathrm{G}$ to $\mathrm{A}$ & Synonymous & Reported polymorphism \\
\hline tRNA histidine & 12192 & $\mathrm{G}$ to $\mathrm{A}$ & & \\
\hline NADH dehydrogenase 5 & 12361 & A to $\mathrm{G}$ & $\mathrm{T}$ to $\mathrm{A}$ & Reported polymorphism \\
\hline NADH dehydrogenase 6 & 14470 & $\mathrm{~T}$ to $\mathrm{C}$ & Synonymous & Reported polymorphism \\
\hline Cytochrome b & 14766 & $\mathrm{C}$ to $\mathrm{T}$ & $\mathrm{T}$ to $\mathrm{I}$ & Reported polymorphism \\
\hline Cytochrome b & 15326 & A to $\mathrm{G}$ & $\mathrm{T}$ to $\mathrm{A}$ & Reported polymorphism \\
\hline Cytochrome b & 15508 & $\mathrm{C}$ to $\mathrm{T}$ & Synonymous & Unreported \\
\hline Cytochrome b & 15662 & A to $\mathrm{G}$ & Synonymous & Unreported \\
\hline Cytochrome b & 15851 & A to $G$ & I to $\mathrm{V}$ & Reported polymorphism \\
\hline tRNA threonine & 15927 & $\mathrm{G}$ to $\mathrm{A}$ & & Reported polymorphism \\
\hline tRNA threonine & 15951 & A to $\mathrm{G}$ & & Reported polymorphism \\
\hline D-loop & 16243 & $\mathrm{~T}$ to $\mathrm{C}$ & & Reported polymorphism \\
\hline D-loop & 16318 & A to $\mathrm{T}$ & & Reported polymorphism \\
\hline D-loop & 16319 & $\mathrm{G}$ to $\mathrm{A}$ & & Reported polymorphism \\
\hline D-loop & 16519 & $\mathrm{~T}$ to $\mathrm{C}$ & & Reported polymorphism \\
\hline
\end{tabular}

Total mtDNA sequencing identified 29 base changes. Twenty-four changes were previously reported polymorphisms and three were unreported but synonymous polymorphisms. The other two changes are G11778A and G12192A

well controlled without serious complications. Echocardiography showed hypertrophic cardiomyopathy with severe loss of compliance and marked thickening of the entire wall of the left ventricle, which continued even after the start of hemodyalysis.

Because of the acute visual loss, LHON was suspected and diagnosed on detection of a G11778A mutation in his mtDNA based on an analysis of restriction fragment length polymorphism. However, because the causes of his clinical manifestations other than visual loss remained unclear, we investigated further.

Genomic DNA was isolated from peripheral leukocytes of the patient using conventional methods. As in a previous study (Akanuma et al. 2001), to avoid nuclear pseudogene amplification, we applied the long polymerase chain reaction (PCR)-based sequencing method. With 96 primer sets designed for sequencing, we sequenced the PCR products using a BigDye Terminator Cycle Sequencing Ready Reaction kit (PE Applied Biosystems, Foster City, CA, USA). The reaction product was then analyzed with an ABI 3700 automated sequencer according to the manufacturer's protocol. The sequence data was compared with MITOMAP (http://www.mitomap.org/mitomap/mitoseq.html).

To identify the G11778A mutation, we amplified the 261-bp PCR fragment and performed an endonuclease $S f a$ NI digestion. In the absence of the mutation, cleaved fragments of 133 and $128 \mathrm{bp}$ were detected. To detect the G12192A mutation, the 174-bp fragment with the mis- matched and reverse primers was digested by $B p 1407$ I. If the fragment had the G12192A mutation, cleaved fragments of 147 and $27 \mathrm{bp}$ could be obtained. Each fragment was detected in a 4\% agarose gel (Nusieve 3:1 agarose; Bio Whittaker Molecular Applications, Rockland, ME, USA) stained with ethidium bromide.

\section{Results}

Total mtDNA sequencing of the patient revealed 29 base changes (Table 1). Twenty-four changes were previously reported polymorphisms and three were unreported but synonymous polymorphisms. The other two changes are thought to be associated with the patient's diseases. One was a G11778A mutation, which is associated with LHON (Fig. 1A), and the other was a G12192A mutation (Fig. 1B). Both mutations were confirmed by restriction fragment length polymorphism, and revealed to be homoplasmic (Fig. 1C, D).

\section{Discussion}

LHON patients have been reported to manifest a variety of ancillary symptoms such as altered reflexes, ataxia, 
Fig. 1.A,B Identification of mutations in the mtDNA. Sequences of polymerase chain reactionamplified products from the mtDNA of the patient, showing A a G-to-A substitution at nucleotide position 11778 (arrow) and B a G-to-A substitution at nucleotide position 12192 (arrow). C, D Detection of G11778A and G12192A mutations. C For the G11778A SfaNI digestion, mutant mtDNA is indicated by a 261-bp band. Wild-type mtDNA is indicated by the presence of a 133- and 128-bp band. D For the G12192A Bp1407I digestion, mutant mtDNA is indicated by a 147-bp band. Wild-type mtDNA is indicated by the presence of a $174-b p$ band. $M$, Molecular weight marker; $P t$, patient; $W T$, wild-type control
A.
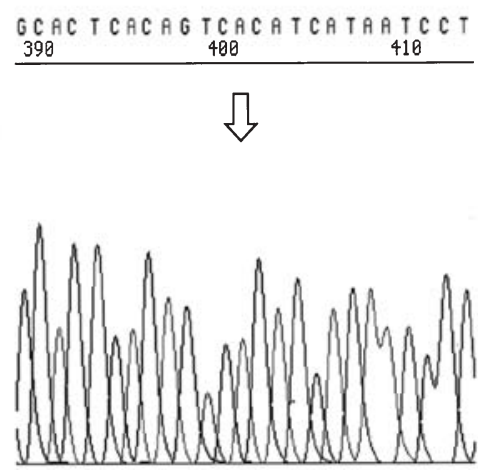

C.
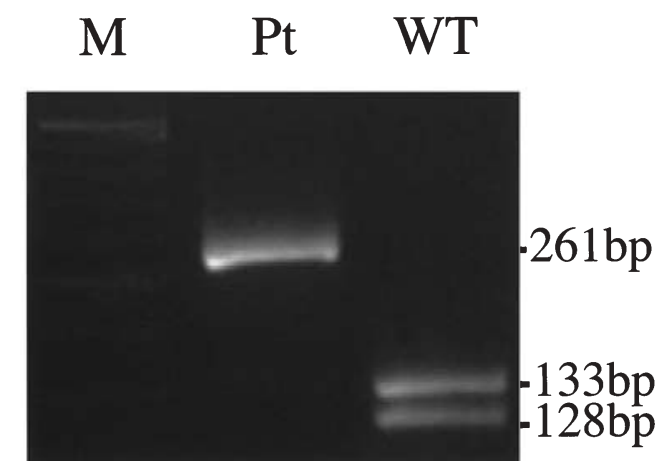

B.

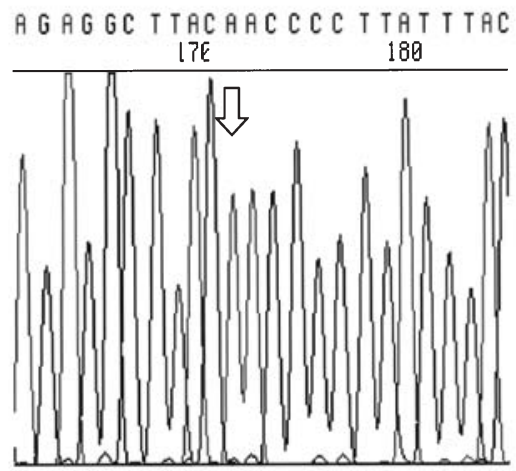

D.
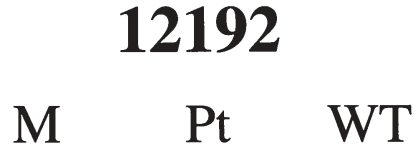

sensory neuropathy, and skeletal abnormalities. Cardiac diseases such as preexcitation syndromes including Wolff-Parkinson-White and Lown-Ganong-Levine (Nikoskelainen et al. 1985) have also been noted in these patients, but there is no report of a patient with cardiomyopathy. Recently, it was shown that a $G \rightarrow A$ substitution at position 12192 in tRNA (His) strongly predisposes carriers to cardiomyopathy (Shin et al. 2000). The second mutation, G12192A, may be related to the additional symptom of hypertrophic cardiomyopathy in the present patient.

This patient also had an $\mathrm{A} \rightarrow \mathrm{T}$ substitution at position 16318 in the D-loop, which was also detected in all five reported patients with cardiomyopathy carrying the G12192A mutation. The fact that the five patients shared this rare single-nucleotide polymorphism suggests a close evolutionary relationship among the mtDNAs of these patients (Shin et al. 2000). On the other hand, phylogenetic analysis of LHON mtDNA has indicated multiple independent occurrences of the G11778A mutation in people with this condition (Brown et al. 1995). According to these findings, one may consider the possibility that the G11778A mutation occurred in a member of a family carrying the G12192A mutation.

Multiple tissue involvement is a key characteristic of mitochondrial diseases with heteroplasmic mtDNA mutations. One patient was reported who carried a heteroplasmic 2532-bp deletion of the type seen in Kearns-
Sayre syndrome, as well as a heteroplasmic A3243G mutation in the tRNA-Leu (UUR) gene of the type seen in cases of mitochondrial myopathy, encephalopathy, lactic acidosis, and stroke-like episodes (Ohno et al. 1996). This patient showed progressive ptosis, ophthalmoparesis, mitochondrial myopathy, pigmentary retinopathy, and autoimmune polyglandular syndrome type II. The two heteroplasmic mutations can be related to these multisystem disorders, but their effects on individual clinical symptoms cannot be separated clearly. Our patient, however, carried two homoplasmic mutations, and the G11778A mutation is in a gene that codes for a protein. To our knowledge, only one male patient with LHON accompanied by chronic renal failure has been reported (Souied et al. 1997) and no cases of LHON accompanied by diabetes mellitus or hypertension have been reported. Thus, it is likely that the effect of the G11778A mutation in this patient is confined to visual impairment typical of LHON, apart from the other symptoms, and it is nearly inconceivable that all of these conditions could be due to smoking and drinking. The G12192A mutations in the tRNA gene may be related to the additional multisystem symptoms, including cardiomyopathy and renal failure accompanied by hypertension and diabetes mellitus.

In several reports, "secondary point mutations" of mtDNA were simultaneously found in LHON patients with "primary mutations," such as the G11778A mutation 
(Matsumoto et al. 1999), which may alone cause LHON. The exact pathogenic role of these mutations however remains unclear and there has been no reported case that includes additional symptoms with which they may be associated. Only two patients carrying the two primary mutations of LHON, G11778A and T14484C, have been reported (Riordan-Eva et al. 1995; Brown et al. 2001). The authors of these reports noted that these mutations might have some influence on the symptoms of LHON, but that they did not cause additional symptoms other than those of LHON.

The present patient suggests the possibility of multiple tissue involvement based on individual polymorphic mutations in mtDNA. To confirm this, further investigations on the separate or synergistic effects of mtDNA sequence on cellular function will be required.

Acknowledgments We thank Mayuko Kato, Yoko Murase, and Munemitsu Yuasa for technical assistance. This work was supported in part by a Research Grant (13B-1) for Nervous and Mental Disorders from the Ministry of Health, Labor and Welfare of Japan (Y.G.).

\section{References}

Akanuma J, Muraki K, Komaki H, Nonaka I, Goto Y (2001) Two pathogenic point mutations exist in the authentic mitochondrial genome, not in the nuclear pseudogene. J Hum Genet 45:337-341

Brown MD, Torroni A, Reckord CL, Wallace DC (1995) Phylogenetic analysis of Leber's hereditary optic neuropathy mitochondrial
DNA's indicates multiple independent occurrences of the common mutations. Hum Mutat 6:311-325

Brown MD, Allen JC, Van Stavern GP, Newman NJ, Wallace DC (2001) Clinical, genetic, and biochemical characterization of a Leber hereditary optic neuropathy family containing both the 11778 and 14484 primary mutations. Am J Hum Genet 104:331-338

Chan DP, Allen HD (1995) Dilated congestive cardiomyopathy. In: Emmanouilides GC, Riemenschneider TA, Allen HD, Gutgesell (eds) Heart disease in infants, children and adolescents, 5th edn. Williams \& Wilkins, Baltimore, pp 1365-1381

Matsumoto M, Hayasaka S, Kadoi C, Hotta Y, Fujiki K, Fujimaki T, Takeda M, Ishida N, Endo S, Kanai A (1999) Secondary mutations of mitochondrial DNA in Japanese patients with Leber's hereditary optic neuropathy. Ophthalmic Genet 20:153-160

MITOMAP: A human mitochondrial genome database (http:// www.mitomap.org/mitomap/mitoseq.html)

Nikoskelainen E, Wanne O, Dahl M (1985) Pre-excitation syndrome and Leber's hereditary optic neuroretinopathy [letter]. Lancet 2:696

Ohno K, Yamamoto M, Engel AG, Harper CM, Roberts LR, Tan GH, Fatourechi V (1996) MELAS- and Kearns-Sayre-type communication with myopathy and autoimmune polyendocrinopathy. Ann Neurol 39:761-766

Riordan-Eva P, Sanders MD, Govan GG, Sweeney MG, Da Costa J, Harding AE (1995) The clinical features of Leber's hereditary optic neuropathy defined by the presence of a pathogenic mitochondrial DNA mutation. Brain 118:319-337

Shin WS, Tanaka M, Suzuki J, Hemmi C, Toyo-oka T (2000) A novel homoplasmic mutation in mtDNA with single evolutionary origin as a risk factor for cardiomyopathy. Am J Hum Genet 67:1617-1620

Souied E, Pisella PJ, Ossareh B, Brezin A, Junes P, Wild-Decrette C, Munnich A, Bonnefont JP, Mondon H (1997) Positive diagnosis of Leber's hereditary optic neuropathy using molecular genetics. J Fr Ophtalmol 20:65-70

Wallace DC, Singh G, Lott MT, Hodge JA, Schurr TG, Lezza AM, Elsas LJ, Nikoskelainen EK (1988) Mitochondrial DNA mutation associated with Leber's hereditary optic neuropathy. Science 242: $1427-1430$ 